\title{
Estudio de la usabilidad web en marcas de moda españolas mediante la herramienta de análisis heurístico Sirius
}

A study of the web usability of Spanish fashion companies using the heuristic-based framework Sirius

Iria Rodríguez

Universidad Nebrija

iria.rodriguezpouso@gmail.com

Guillermo de Haro

Universidad Rey Juan Carlos (URC)

guillermo.deharo@urjc.es

Ignacio Somalo

IE Business Schoo

nacho.somalo@gmail.com
Rodríguez, I., de Haro, G, y Somalo, I. (2017)

Estudio de la usabilidad web en marcas de moda españolas mediante la herramienta de análisis heurístico Sirius

Revista Internacional de Investigación en Comunicación aDResearch ESIC. № 15 Vol 15

Primer semestre, enero-junio 2017 · Págs. 8 a 31

DOI: 15.7263/ADRESIC.015.001 
RESUMEN

Clasificación JEL:

M32

Palabras clave:

expericiencia de

usuario,

usabilidad,

comercio electrónico,

moda,

valor de marca

\section{ABSTRACT}

JEL Classification:

M32

Key words:

user experience,

usability,

ecommerce,

fashion,

brand value
Actualmente es complicado encontrar una empresa que no tenga como parte de su estrategia la presencia web. El impacto de la gestión de la interacción entre los usuarios y la tecnología web (usabilidad) es de vital importancia y puede ser la base para mejorar posteriormente la gestión de la marca o tomar decisiones de comercio electrónico. Este estudio busca realizar un análisis de evaluación sin usuarios con la herramienta heurística Sirius de la usabilidad web de las principales marcas de moda españolas. Seleccionando dichas marcas a partir del informe Interbrand se estudian las webs de marcas como Zara, Mango o Desigual, analizando diferentes aspectos de su usabilidad web.

These days it is not easy to find a company which does not have a website as part of its business strategy. The impact of the management of interaction between users and web technology (usability) is of vital importance and can be a starting point to improve brand management or make decisions about electronic commerce. The aim of this study is to carry out an evaluative analysis, without users and using the heuristic-based framework Sirius, on the website usability of the most important Spanish fashion companies. These companies were selected from the Interbrand report and websites belonging to brands such as Zara, Mango and Desigual are studied, analyzing different aspects of web usability. 


\section{Introducción}

El presente trabajo se plantea como el punto de partida para un estudio más amplio de la usabilidad digital en España. En él se pretende identificar qué elementos de la usabilidad están más y menos cuidados en algunas de las marcas más relevantes de nuestro país, de tal modo que la aplicación de mejoras en la usabilidad esté dirigida a aquellos aspectos menos tenidos en cuenta, y que pueden mejorar de forma notable la experiencia de usuario así como impactar a futuro en otros aspectos más relevantes como la gestión de marca.

El concepto de usabilidad no está definido en el diccionario de la Real Academia Española (RAE). Es sin embargo de uso habitual en diferentes entornos como la informática y la tecnología, al ser un neologismo proveniente del inglés «usability» o facilidad de uso. La usabilidad es representada desde una disciplina mucho mayor conocida como interacción persona-ordenador. Bajo dicha disciplina se han ido desarrollando diversas ciencias y tecnologías que en sus inicios estaban supeditadas únicamente a ámbitos especializados, siendo por lo tanto diseñadas para usuarios expertos. Por ejemplo el libro «Medical Devices. Guidance on the Application of Usability Engineering to Medical Devices», publicado en 1916 por el British Standards Institute, ya apuntaba la relevancia del concepto en un entorno especializado, en este caso las ciencias de la vida.

Con el paso del tiempo se han definido diferentes niveles de acceso y se ha logrado extender su uso a ámbitos y entornos muy variados (Ortega, 2013).

El estándar ISO 9241-171 de 2008 define la usabilidad como «el grado en que un producto puede ser utilizado por usuarios especificados para lograr objetivos concretos con eficacia, eficiencia y satisfacción, en un determinado contexto de utilización».

La usabilidad digital, según Nielsen $(2003)^{1}$, se centra en la facilidad de uso de las diferentes aplicaciones interactivas. A la hora de comentar la usabilidad como un atributo de calidad es necesario tener en cuenta al usuario y su experiencia. De este modo Nielsen valora diferentes variables para su medición:

- La facilidad de aprendizaje de los usuarios, teniendo especial relevancia la primera vez que éstos se enfrentan a un nuevo diseño y deben llevar a cabo una serie de tareas. Esta primera vez es clave, si el manejo es intuitivo existe la posibilidad de que el usuario repita su experiencia.

- La eficiencia de las aplicaciones para que los usuarios lleven a cabo diferentes tareas. Una vez los usuarios han aprendido el manejo de la aplicación digital, es necesario que comiencen a darle uso realizando una serie de tareas. El tiempo que tardan en realizarlas es primordial para conocer si la eficiencia de la aplicación digital es la óptima o no.

- La cualidad de ser recordado en el momento en que los usuarios acceden de nuevo a la aplicación. El tiempo que tardan en volver a adquirir el conocimiento necesario para dar de nuevo un uso eficiente a las aplicaciones indicará el nivel de intuición de la misma.

- La eficacia de una aplicación se observará en el momento en que los usuarios están realizando las tareas para que se ha diseña-

\footnotetext{
1 Para Nielsen, 2003, la usabilidad juega un papel en cada etapa del proceso de diseño. La necesidad resultante de múltiples estudios es una razón por la que recomienda la realización de estudios individuales, además cuentan con la ventaja de que son rápidos y económicos.
} 
do ésta. Se observan los errores que se cometen, la gravedad y la frecuencia de los mismos, así como si el usuario es capaz de realizar las correcciones necesarias.

- La satisfacción de los usuarios también es un elemento a tener en cuenta a la hora de medir la usabilidad, ya que observar la comodidad del usuario en el momento de realizar las tareas necesarias, así como si su percepción general de las mismas ha sido agradable y sencilla.

En este estudio nos centraremos en la medición de la usabilidad digital enfocada a la interacción con las páginas web de las diferentes marcas objeto del estudio.

\section{La usabilidad. Un atributo relevante para un producto digital}

En sus inicios las interfaces de usuario se constituían de grandes paneles repletos de instrumentos, y en constante supervisión por varios operadores. Posteriormente se introdujeron sistemas de supervisión distribuida y trabajo cooperativo, lo que ha implicado la entrada del ordenador de forma masiva en las empresas y, por ende, las interfaces gráficas de usuario o interfaces hombre-ordenador (Moreno, 2003).

Dentro de ISO se definen cuatro categorías de estándares de usabilidad (Alan et al., 2003), cada una con definiciones diferentes:

- Capacidad organizativa: Capacidad de uso, ISO 18520. Relacionada con el ciclo de vida del proceso.

- Calidad del proceso: Proceso centrado en el usuario, ISO 13407. Relacionada con el desarrollo de procesos.

- Calidad del producto: Interfaces e interacciones, ISO 9241 partes 10, 12-17, ISO/IEC 9126-3. Relacionada con los productos.
- Usabilidad en contexto: Calidad de uso, ISO 9241-11, ISO 14598-1, ISO/IEC 9126 1,4. Efecto del producto.

Por lo tanto tenemos dos grandes categorías de estándares: por una parte los estándares orientados al producto y por otra parte los estándares orientados al proceso. Dentro de estos podemos comprobar como la usabilidad tiene en cuenta diversos factores a la hora de diseñar productos usables. "Los usuarios no buscan usabilidad, buscan utilidad, entendida como el provecho, beneficio e interés que produce su uso.» (Hassan, 2006: 247). La definición de usabilidad bajo ISO/IEC 9126-1 (2000) sería «la capacidad de un producto software para ser entendido, aprendido, utilizado y atractivo para el usuario, cuando se usa bajo ciertas condiciones». Sin embargo bajo ISO 9241-11 (1998) «el punto hasta el que un producto se puede utilizar por determinados usuarios para alcanzar determinados objetivos con eficacia, eficiencia y satisfacción dentro de un contexto de uso determinado». Un último ejemplo sería la definición de IEEE en su estándar 610.12 (1990) «la facilidad con la que un usuario puede aprender a operar, preparar insumos para e interpretar resultados de un sistema o componente». Comprobamos por tanto cómo además debemos tener en cuenta la diferencia entre usuario y sistema para poder entender la interacción entre ambos, tanto a nivel de proceso como de producto.

Si un sitio web no satisface las necesidades de los usuarios difícilmente lo hará con las necesidades que la organización quiere cubrir al proporcionarlo (Bevan, 1997). Algunas de las principales dificultades para los usuarios para encontrar contenido en un sitio web son que las organizaciones producen contenidos similares a los que utilizan internamente, sin tener en cuen- 
ta el entorno web ni el tipo de usuario que accederá al contenido; que a menudo utilizan material sin adaptar al formato web; o que los criterios de calidad se miden con los sistemas tradicionales de las organizaciones a la hora de generar contenidos.

Para Hassan y Ortega (2009) la verdadera naturaleza de la usabilidad radica en cuatro dimensiones diferentes, de tal modo que establecen una definición de la usabilidad más completa:

- Dimensión empírica. Como concepto empírico la usabilidad puede ser medida y evaluada. Desde un punto de vista de calidad Hassan y Ortega consideran relevantes las variables que distingue Nielsen (2003) a través de las cuales puede ser medida (facilidad de aprendizaje, eficiencia, facilidad para ser recordado, eficacia, satisfacción). La naturaleza empírica de la usabilidad da la posibilidad de modificar y adaptar un proyecto de diseño teniendo en cuenta los resultados de las métricas.

- Dimensión dependiente. Existe una relación entre utilidad y usabilidad de mutua dependencia. La relevancia de la utilidad percibida es el nivel en el que un usuario cree que el uso del sistema mejorará su rendimiento, por lo que representa una conexión directa con la usabilidad y, por ende, con la aceptabilidad del producto.

- Dimensión relativa. Para que un producto sea usable, éste debe satisfacer las necesidades de sus usuarios. Es por ello que la usabilidad tiene una estrecha relación con los objetivos, el contexto de uso o las tareas que se desarrollen.

- Dimensión ética. El objetivo de los productos no es simplemente el de satisfacer las ne- cesidades de sus usuarios, sino también la mejora de su calidad de vida. Para ello es básico una interacción constante con los usuarios, empatizar con ellos o la experimentación de los productos en primera persona.

Por lo tanto, para definir y medir la usabilidad digital, debemos tener en cuenta aspectos como la accesibilidad, la arquitectura de la información, el diseño de la interacción, el diseño gráfico, la simplicidad, la navegabilidad y la experiencia de usuario que funciona como integradora de todos los elementos.

La accesibilidad de un producto digital se define como «la posibilidad de que pueda ser accedido y usado por el mayor número posible de personas, indiferentemente de las limitaciones propias del individuo o de las derivadas del contexto de uso». (Hassan y Martín, 2004: 332). En el diseño de aplicaciones digitales se debe tener en cuenta a todas las personas, independientemente de sus capacidades, tanto de hardware o software, así como capacidades físicas o mentales. La accesibilidad se integra con el diseño de los smartphones, el diseño para usuarios con más edad, y la optimización para motores de búsqueda (Berners-Lee, 2013).

La arquitectura de la información se basa en organizar, clasificar, estructurar y describir los contenidos digitales. De este modo los usuarios se podrán informar con el menor esfuerzo posible. La arquitectura de la información se representa como el esqueleto de una aplicación digital, por lo tanto es uno de los puntos que deben definirse en el comienzo de los proyectos digitales (Hassan y Ortega, 2009: 12). Una mala arquitectura de la información es la piedra con la que tropiezan los usuarios cuando buscan cualquier tipo de contenido, por lo tanto les impide llegar a realizar otras tareas (Nielsen, 2009). 
La evaluación de la usabilidad es un área compleja desde el momento en que la medición de la usabilidad depende de cómo se defina usabilidad (Jordan et al., 1996). Y dentro de este proceso de definición es imposible especificar el valor de la usabilidad de un sistema, o dicho de otro modo cómo cumple con su función, sin determinar quienes van a ser los usuarios del mismo, las tareas que deben realizar con el sistema así como los principales aspectos del entorno (organizativo, social u otros) en que será utilizado.

La experiencia de usuario se diseña en función del contexto, del contenido y de los propios usuarios, estos tres elementos funcionan relacionados entre sí. En el proceso de creación de una aplicación digital, las decisiones en cuanto a diseño variarán en función de estos elementos (Morville, 2004).

Para D'Hertefelt (2000), la experiencia de usuario implica un cambio sustancial del concepto de usabilidad, el objetivo de ésta no es únicamente la mejora el rendimiento del usuario en la interacción (eficacia, eficiencia y facilidad de aprendizaje), va más allá intentando resolver el problema estratégico de la utilidad del producto y el problema psicológico del encanto y diversión de su uso:

- Problema estratégico: busca conseguir que los productos sean útiles y usables.

- Problema psicológico: busca conseguir que los productos sean aceptados por los usuarios.

Por otro lado, la experiencia de usuario tiene en cuenta otra serie de elementos con los que se va trabajando a medida que evoluciona el proyecto de diseño de aplicaciones digitales. La concepción comenzaría definiendo los objetivos de sitio, las necesidades del usuario, avanzaría con los contenidos, la arquitectura de la información, y culminaría con el diseño, el diseño de la información, el diseño de la navegación y finalmente el diseño visual (Garrett, 2000).

La usabilidad en productos digitales es un atributo relacionado con la facilidad de uso. Es relevante en este sentido la rapidez en la que los usuarios aprenden a utilizar algo, la eficiencia al utilizarlo, cuan memorable es, cuál es su grado de propensión al error y cuánto les gusta a los usuarios. Si una característica u opción en la web no se puede utilizar o no se utiliza, es como si no existiera. Una buena usabilidad en un sitio web tiene dos ventajas: por un lado, respalda sus objetivos empresariales en la propia web y ayuda de esta forma a la compañía a obtener mayores beneficios sean o no monetarios (Nielsen y Loranger, 2006).

En 1993 Nielsen manifestaba que la utilidad de un sistema, entendida ésta como un medio con el que lograr un objetivo, dispone de dos componentes, por un lado, uno basado en la funcionalidad y, por otro lado, uno basado en la forma en la que los usuarios son capaces de usar esta funcionalidad. Por otra parte en el mismo año se propone un modelo de usabilidad basado en la eficacia, eficiencia, satisfacción y facilidad de aprendizaje (Dix et al, 1993), que Nielsen adapta posteriormente manteniendo las mismas variables principales (Nielsen, 1994).

La llegada de la tecnología móvil ha supuesto un nuevo reto en esa disciplina. Existen diferentes estudios sobre el impacto de la tecnología móvil en la usabilidad. Se ha relacionado de manera empírica la usabilidad con la lealtad de marca, a través de los constructos de satisfacción y confianza (Lee at al, 2015), así como la importancia de la adaptación del diseño web a las pantallas de los dispositivos móviles y su impacto en 
la percepción de uso, permanencia en página y experiencia general de consumo del contenido (Yu y Kon, 2016).

En la actualidad las marcas están influidas a la vez que definidas por sus audiencias, y en especial lo que éstas demandan. La gran velocidad a la que evoluciona el mundo implica una mayor difusión e interacción entre medios y plataformas, pues cada vez son menores las diferencias entre lo virtual y lo real. Es por ello que las marcas son la experiencia que ofrecen a sus consumidores, ya sea a través de su web, sus tiendas o sus envases (Borrero, 2014). Por lo tanto entender y valorar su usabilidad web es un primer paso primordial para poder valorar posteriormente su impacto en el diseño y gestión de marcas.

Así que a pesar de la extensa literatura sobre usabilidad en general existe sin embargo todavía una importante laguna en lo relativo a entender con precisión y de manera específica la usabilidad web y su impacto en relación con otros aspectos del marketing (Islam y Bouwman, 2016). Símbolos como enlaces de navegación, pequeñas imágenes, botones o similares pueden ser analizados desde perspectivas tecnológicas, semióticas y otras más, para su posterior aplicación a la toma de decisiones en la empresa. El problema no es nuevo. Desde la propia definición de «calidad de producto» (Garvin, 1984) se planteaba el problema de la cobertura de una temática que abarcaba disciplinas como economía, marketing, gestión de las operaciones o incluso filosofía. Un problema similar se plantea con la usabilidad.

\section{Metodología}

Este análisis toma como base el último ranking bianual que la consultora Interbrand realiza sobre marcas españolas. Además del modelo de evaluación heurística sobre usabilidad: Sirius, presentado por María del Carmen Suárez Torrente en su Tesis Doctoral en 2011.

Evaluar la usabilidad es una de las tareas más relevantes de cara a emprender el desarrollo de una interfaz. Pues una interfaz pobre puede frustrar a los usuarios, de tal modo que en un mercado competitivo éstos sean conducidos hacia los competidores (Woodward, 1998).

Por lo tanto, uno de los objetivos que tiene en cuenta el propósito de evaluar la usabilidad es, el de valorar en qué medida se están consiguiendo cumplir los objetivos marcados frente a los usuarios y a la propia organización (Suárez, 2011: 25).

Calvo-Fernández et al, 2013 y Zapata, 2013 diferencian dos tipos de métodos para evaluar la usabilidad. Métodos de evaluación con usuarios y métodos de evaluación sin usuarios:

\footnotetext{
Métodos de evaluación sin usuarios

- Evaluación heurística

- Recorrido o paseo cognitivo

- Inspecciones formales

- Inspecciones de características

- Inspecciones de consistencia

- Inspecciones de estándares

- $\mathrm{GOMS}^{2}$
} 
Zapata, $2013^{3}$, realiza una radiografía de los diferentes métodos de evaluación digital sin usuarios:

\section{El recorrido cognitivo}

El recorrido cognitivo es una técnica de revisión en la que los evaluadores expertos de la usabilidad construyen escenarios para las distintas tareas que necesitan evaluar sobre el sistema, de tal modo que posteriormente a la evaluación, éstos puedan emular al usuario trabajando con la interfaz.

Los evaluadores deben interpretar si las tareas son adecuadas o no en función de su experiencia y conocimiento previo sobre el comportamiento y reacciones de los usuarios, es por ello, que este método lleva implícito un posible porcentaje de error de interpretación.

Este método tiene sus orígenes en Lewis y Polson (1990) y en el cognitive jogthrough de Rowley y Rhoades (1992), obtuvo su máxima popularidad como técnica de inspección de la usabilidad cuando Wharton, Rieman, Lewis y Polson lo describieron en The cognitive walkthrough method: a practitioner's guide (Nielsen y Mack, 1994).

\section{Inspecciones formales de usabilidad}

Las inspecciones formales de usabilidad tienen su base en la metodología de inspección del software, donde ésta es adaptada a la evaluación de la usabilidad.

Las inspecciones de usabilidad han sido estudiadas minuciosamente por Kahn y Prail en Usability inspection methods (Nielsen y Mack, 1994). Una de sus principales características es que incluye aspectos de otros métodos de inspección.

3 El estudio de Zapata, 2013, es una inspección teórica de los diferentes métodos de evaluación de usabilidad sin usuarios.
Los principios heurísticos están reflejados como elemento de apoyo para la búsqueda de errores. Y los evaluadores recorren meticulosamente las tareas con los propósitos y objetivos de los usuarios en mente de forma similar a los recorridos cognitivos, aunque el énfasis radica menos en la teoría cognitiva y más en el hallazgo de errores.

\section{Inspecciones de características}

La inspección de características, se ha descrito en Usability inspection methods (Nielsen y Mack, 1994), y se centra en el análisis de ciertos elementos de un sistema preferiblemente en las etapas intermedias de su desarrollo.

El objetivo de la inspección es asegurar la calidad final del sistema o interfaz, por ello es importante que previamente se hayan descrito cuáles son las características que éste debe contener.

\section{Inspecciones de consistencia}

En las inspecciones de consistencia, también descritas por Kahn y Prail en Usability inspection methods (Nielsen y Mack, 1994), el objetivo principal radica en la identificación de inconsistencias entre contextos de interacción y sus funcionalidades o contenidos.

Por lo tanto, los evaluadores o profesionales de la usabilidad deben analizar las interfaces de todos los sistemas y detectar inconsistencias en cuanto a terminología, color, disposición de los elementos en la pantalla, formatos de entrada y salida de datos, entre otros.

\section{Inspecciones de estándares}

Las inspecciones de estándares, comentadas por Dennis Wixon también en Usability inspection methods (Nielsen y Mack, 1994), garantizan el ajuste a los estándares, entendiendo por estándar una especificación que regula la realización de 
ciertos procesos o la fabricación de componentes para garantizar la interoperabilidad.

Los estándares de iure, son aquello que han sido generados por un comité con estatus legal y que gozan del apoyo de un gobierno o una institución para producir estándares ${ }^{4}$.

Los estándares de facto son patrones o normas que se caracterizan por no haber sido consensuados ni legitimados por un organismo de estandarización al efecto, aunque se trata de normas generalmente aceptadas y ampliamente utilizadas por iniciativa propia de un gran número de interesados.

Por este motivo, los estándares de facto son la antítesis de los estándares de iure. Pues su descripción se encuentra normalmente en lugares como los manuales, libros y artículos y son aceptados como tales por su uso generalizado. De todos modos, algunos estándares de facto acaban derivando en estándares de iure.

\section{GOMS}

GOMS es un modelo hipotético de cómo se realizan las cosas y permite predecir la duración de una tarea específica. De hecho, el modelo GOMS permite entender la forma de como las personas interactúan con las máquinas, definir los métodos de trabajo, seleccionar los procedimientos y calcular tiempos y velocidades para completar eficazmente determinadas metas.

Card, Moran y Newell (1983) llevaron a cabo la formulación original de GOMS, además crearon una versión simplificada, el modelo de pul-

4 Algunos de los organismos con estatus legal son:

- Asociación Internacional de Estándares (ISO).

- El Instituto Nacional Americano de Estándares (ANSI).

- El Instituto de Ingenieros Eléctricos y Electrónicos americano (IEEE)

- El Comité Europeo para la Estandarización (CEN).

- El World Wide Web Consortium (W3C). saciones de teclas KLM (keystroke-level model). Otros autores, basándose en el mismo concepto de GOMS, desarrollaron otras versiones como CMN-GOMS y NGOMSL (natural GOMS language), aunque una de las más conocidas es KLM-GOMS, que se basa en analizar las tareas y describirlas, teniendo en cuenta las operaciones elementales, como puede ser una pulsación de teclado o el movimiento del ratón.

La evaluación de la usabilidad es una parte relevante e integrada en el desarrollo de un producto interactivo. Una manera óptima de realizar esta evaluación es a través de un proceso iterativo de tal modo que puedan alcanzar los objetivos mercados de manera progresiva. Todos estos estudios iniciales, pruebas y prototipos forman parte de un proceso más amplio, que valora la capacidad para obtener un producto con el que los usuarios se sientan cómodos y que perciban que su uso les va a facilitar la consecución con éxito de una tarea (Ortega, 2013) 5 .

Wixon y Wilson (1997: 681) muestran varias dimensiones en las que los métodos de usabilidad se pueden clasificar. Estos pueden ser considerados como la creación de un espacio multidimensional. En teoría, cada dimensión es lógicamente independiente, pero en la práctica los métodos tienden a agruparse en ciertas áreas definidas por estas dimensiones:

- Formativa en comparación con la sumativa. Los métodos de formativos se utilizan para crear un diseño. Por ejemplo, una lluvia de ideas o los guiones gráficos son métodos formativos. Los métodos sumativos se utili-

\footnotetext{
5 Para Ortega (2013) la usabilidad es un elemento cambiante, sujetos a diversas variables que en cada contexto adquieren un significado diferente. Es por ello que, para la mejora de la calidad de un producto interactiva, es necesario la mejora de su uso, la búsqueda de nuevos enfoques y de diferentes perspectivas.
} 
zan para evaluar un diseño. Por ejemplo, las evaluaciones comparativas y encuestas de satisfacción son métodos sumativos.

- Métodos de descubrimiento frente a métodos de decisión. Los métodos de descubrimiento se utilizan para descubrir la forma que trabajan los usuarios, como se comportan, como piensan o qué problemas tienen. Los métodos de descubrimiento también están orientados hacia la tecnología. Los métodos de decisión se utilizan para ordenar o elegir entre los elementos de la interfaz o los diseños completos.

- Métodos formalizados frente a métodos informales. Se adaptan a las necesidades de una situación.

- Métodos con usuarios involucrados en comparación a métodos sin usuarios. Los métodos difieren en la medida en que los usuarios están implicados en la evaluación, el análisis y el diseño.

- Métodos completos frente a los métodos de componentes. Algunos métodos cubren todos los pasos necesarios para completar el trabajo de diseño de usabilidad. Otros métodos son métodos de componentes que representan una parte de un proceso de la facilidad de uso completo.

La evaluación heurística es un método de evaluación de la usabilidad por inspección, se realiza por expertos en usabilidad a partir de unos principios establecidos por la disciplina de la interacción persona-ordenador (IPO), también conocidos como principios heurísticos ( $\mathrm{Za}$ pata, 2013: 7).

Para Calvo-Fernández et al, 2013, las evaluaciones heurísticas pueden llevarse a cabo bien sobre la totalidad de una interfaz, bien evaluan- do tan sólo secciones concretas. Además, también puede abordarse a dos niveles de profundidad:

- nivel alto: en aquellos casos en los que interese centrarse en la evaluación de ciertas tareas o procesos o bien en detectar los principales errores de la interfaz en términos genéricos.

- nivel bajo: en aquellos casos en los que interese bajar al detalle de cada una de las páginas o pantallas de la interfaz.

Respecto a cuándo llevar a cabo una evaluación heurística, ésta se tiene en cuenta en distintas etapas de un proyecto:

- En fases iniciales, permitiendo trabajar con interfaces aún no implementadas, testando prototipos y buscando aquellos puntos que pueden ser mejorados.

- Durante el desarrollo, realizando revisiones para localizar y corregir errores y fallos, lo que permite solucionar problemas con un coste menor que si se detectan en la fase final de desarrollo o una vez finalizado el producto.

- En productos, aplicaciones o sitios web ya existentes.

Este método de evaluación fue desarrollado por Molich y Nielsen en $1990^{6}$. De tal modo que con él se evalúan los elementos de una interfaz con el objeto de medir su calidad con relación a la facilidad para ser aprendido y usado. Por lo

\footnotetext{
6 Para Nielsen y Molich, 1990, la evaluación heurística se realiza observando una interfaz y tratando de llegar a una opinión sobre lo que es bueno y malo de la misma.

Lo ideal es que estas evaluaciones se llevasen a cabo teniendo en cuenta ciertas reglas. La lista de heurísticas propuesta por Nielsen y Molich ha sido desarrollada durante varios años teniendo en curta su experiencia, tanto en la enseñanza, como en la consultoría sobre usabilidad.
} 
tanto, la evaluación heurística busca resultados cualitativos que den la posibilidad de resaltar los problemas de usabilidad de las interfaces.

La definición que ofreció Pearl en 1984 posiblemente es la que más se aproxima a la que se maneja hoy en día. «Los heurísticos son criterios, métodos o principios que nos ayudan a decidir, entre varias acciones, cuál promete ser la más eficaz para lograr algún objetivo. Representan un compromiso entre dos exigencias: la necesidad de marcar criterios simples y, al mismo tiempo, el deseo de discriminar correctamente entre opciones buenas y malas.» (Pearl, 1984, pág. 31).

Nielsen (1995) propuso diez heurísticos entendidos como guía para el diseño y evaluación de la usabilidad.

- Visibilidad del estado del sistema: El sistema siempre debe mantener a los usuarios informados acerca de lo que está pasando, a través de la retroalimentación adecuada en un tiempo razonable.

- Relación entre el sistema y el mundo real: El sistema debe hablar el idioma de los usuarios, con palabras, frases y conceptos familiares para el usuario, en lugar de términos orientados al sistema. Se deben seguir las convenciones del mundo real, haciendo que la información aparezca en un orden lógico y natural.

- Control del usuario y libertad: Los usuarios a menudo eligen funciones del sistema por error y necesitarán que se les muestre claramente como deshacer y rehacer.

- Consistencia y estándares: Los usuarios no deberían tener que preguntarse si diferentes palabras, situaciones o acciones significan lo mismo.
- Prevención de errores: Un diseño bien cuidado, evita que se produzcan errores. Es necesario por lo tanto eliminar las condiciones propensas a los mismos.

- Reconocimiento en vez de recordar: Reducir al mínimo la posibilidad de que el usuario tenga que recordar la información. Las instrucciones de uso del sistema deben ser visibles o fácilmente recuperables cuando sea necesario.

- La flexibilidad y la eficiencia del uso: Los aceleradores a menudo pueden acelerar la interacción para el usuario experto de tal manera que el sistema puede servir tanto a los usuarios inexpertos y experimentados. En necesario permitir a los usuarios personalizar las acciones frecuentes.

- Diseño estético y minimalista: Los textos no deben contener información que es irrelevante o poco necesaria. Cada unidad adicional de información en un texto compite con las unidades pertinentes de información y disminuye su visibilidad relativa.

- Ayudar a los usuarios a reconocer, diagnosticar y recuperarse de los errores: Los mensajes de error deben expresarse en un lenguaje sencillo (sin códigos), es necesario indicar con precisión el problema y sugerir una solución constructiva.

- Ayuda y documentación: A pesar de que es mejor si el sistema puede ser utilizado sin la documentación, puede ser necesario proporcionar ayuda y documentación. Dicha información debe ser fácil de buscar, centrarse en la tarea del usuario, y no ser demasiado extensa.

Suárez (2011) en su Tesis estudia y evalúa los principales métodos heurísticos desarrollados 
desde que Molich y Nielsen presentaran el suyo en 1990. Asimismo, no todos los métodos son considerados evaluaciones heurísticas como tal, sino que se presentan en forma de principios generales de usabilidad. Como conclusión al estudio de todos estos métodos, Suárez plantea una falta de acuerdo en cuanto a la utilización de unas pautas estandarizadas y una falta de elementos de valoración común. Con el fin de solventar estas carencias desarrolla un nuevo sistema de evaluación heurística

El sistema Sirius desarrollado por Suárez ofrece la posibilidad de conocer el nivel de evolución de un sitio web en un periodo de tiempo, así como comparar dos o más sitios web respecto a su nivel de usabilidad y elaborar rankings de usabilidad en base a unos criterios uniformes.

Suárez plantea en su sistema una serie de aspectos a considerar para la evaluación de la usabilidad en un sitio web. El valor que obtiene cada uno de estos aspectos será ponderado en función del tipo de sitio web. Bien es cierto que cada tipo de sitio web dispone de unos objetivos diferentes, por lo que el valor de cada aspecto variará en función de esta tipología.

La relación de aspectos que se valoran en el sistema Sirius son los siguientes:

- Aspectos generales: Aquellos elementos relativos a los objetivos del sitio web como su look E feel, coherencia y nivel de actualización en los contenidos.

- Identidad e Información: Aquellos elementos relacionados con la identidad del sitio, la información que se proporciona sobre los autores de contenidos del mismo o la corporación a la que pertenece.

- Estructura y Navegación: Aquellos elementos relacionados con la idoneidad de la arquitectura de la información del sitio web, así como la navegación del mismo.

- Rotulado: Aquellos elementos relacionados con la trascendencia, corrección, y familiaridad del rotulado de los contenidos.

- Layout de la página: Aquellos elementos relativos a la distribución y el aspecto de los elementos de la interfaz.

- Entendibilidad y facilidad en la interacción: Aquellos elementos que tienen relación con la calidad de los contenidos de texto, los iconos y los controles de la interfaz.

- Control y retroalimentación: Aquellos elementos relacionados con la libertad de navegación que dispone el usuario, así como la información que se le proporciona en la interacción con el sitio web.

- Elementos multimedia: Aquellos elementos relacionados con los contenidos multimedia que están presentes en el sitio web.

- Búsqueda: Aquellos elementos relaticos al buscador que el sitio web pone a disposición del usuario.

- Ayuda: Aquellos elementos que tienen relación con la ayuda que se le ofrece al usuario durante su navegación en el sitio web.

El sistema Sirius trata de desarrollar un sistema de evaluación heurística que pretende solventar las carencias debidas la gran cantidad de heurísticas o pautas de diseño existentes. Además, no se cuenta tampoco con un esquema de organización de las mismas que permita su clasificación y búsqueda; Suárez constata también que existe muy poca coherencia entre las diferentes propuestas

Para el desarrollo del modelo, en primer lugar, se establece una clasificación de sitios web para poder abordar las siguientes tareas: 
- Adaptar los resultados de la evaluación de un sitio a la categoría del mismo, ponderando los valores obtenidos en función del tipo de sitio considerado.

- Determinar las tareas que para cada tipo de sitio se consideran críticas y el perfil de usuario más habitual del sitio. De esta manera, esta relación de tareas propias de cada tipo de sitio podrán ser consideradas en la etapa de evaluación con usuarios, realizando la evaluación con todos los perfiles obtenidos.

La clasificación de sitios web que propone Suárez es la siguiente:
En su sistema de evaluación Suárez establece una única relación de elementos a evaluar denominados criterios (subheurísticas), agrupados en aspectos (heurísticas) que será utilizada por todos los expertos implicados en el proceso de evaluación. Con una única relación de elementos a evaluar se consigue unificar los criterios para llevar a cabo una evaluación heurística de un sitio web.

Perurena y Moráguez realizan en 2013 un análisis de diferentes técnicas y métodos de evaluación de usabilidad web, con el objetivo de validar el sistema SIRIUS. En sus conclusiones se verifica como un método para establecer clasifi-
- Administración Pública / Institucional

- Banca electrónica

- Blog

- Comercio electrónico

- Comunicación / Noticias

- Corporativo/Empresa
- Descargas

- Educativo / Formativo

- Entornos colaborativos/Wikis

- Foros/Chat

- Ocio/Entretenimiento

- Personal
- Portal de servicios

- Servicios interactivos basados en imágenes

- Servicios interactivos no basados en imágenes

- WebMail/Correo
Una vez establecida la relación de los tipos de sitios a considerar en el sistema de evaluación se ha obtenido para cada tipo de sitio la audiencia habitual de cada uno de ellos y la relación de tareas críticas o relevantes en el mismo. El objetivo principal de la determinación de la audiencia y tareas críticas es la de facilitar el diseño de las pruebas de evaluación. Para Suárez una evaluación basada en el usuario se puede analizar un subconjunto de todas las tareas que soporta el sistema, la evaluación debe estar basada en el análisis de las tareas más representativas escogidas por su frecuencia o criticidad. caciones o rankings en base al criterio de usabilidad, conocer la evolución de la usabilidad en los sitios web a lo largo del tiempo, y permitir realizar estudios de relación entre la usabilidad y otras variables como la accesibilidad.

Para establecer tanto los aspectos como los criterios en el sistema de evaluación Sirius, Suárez ha considerado la experiencia y el conocimiento relativo a métodos de evaluación heurística propuesta por diversos expertos en evaluación de usabilidad.

La relación de aspectos que se valoran en el sistema Sirius son los siguientes:
- Aspectos generales

- Identidad e Información

- Estructura y Navegación

- Rotulado
- Layout de la página

- Entendibilidad y facilidad en la interacción

- Control y retroalimentación
- Multimedia

- Búsqueda

- Ayuda 
Cada uno de los aspectos enumerados anteriormente se concreta en una serie de criterios evaluables a los que el evaluador deberá asignarles un valor durante el proceso de evaluación. Por lo tanto, el evaluador únicamente se ocupará de cuantificar el incumplimiento, no la severidad o relevancia del mismo. Además, se ha determinado el nivel de relevancia del incumplimiento de un aspecto en relación a un tipo de sitio, se ha ponderado también, el nivel de importancia del incumplimiento (o severidad) de cada criterio en relación al tipo de sitio que se esté evaluando.

En último lugar, Suárez considera necesario obtener un valor cuantitativo que refleje el nivel de usabilidad obtenido tras la evaluación heurística de un sitio web. Para ello se propone una fórmula en la que se consideran los siguientes conceptos:

- Factor de corrección: Valor de ajuste que se aplica a cada uno de los criterios evaluados con el fin de obtener diferentes niveles de usabilidad dependiendo de la relevancia de los mismos en función del tipo de sitio web en evaluación. Los valores resultantes al aplicar este factor de corrección debieran llevar a obtener valores de usabilidad comprendidos siempre entre 0 y 100, por tratarse de un porcentaje.

- Cálculo del Factor de Corrección: partiendo de los valores correspondientes a los diferentes niveles de relevancia de un criterio, el factor de corrección se calcula dividiendo cada valor de relevancia entre la suma de todos los valores de relevancia de los criterios evaluados.

Finalmente, Suárez en la propuesta de su modelo ha realizado una serie de experimentos, que le han permitido, por un lado, ajustar los valores de relevancia de los criterios planteados inicialmente. Y, por otro lado, contrastar la validez del sistema propuesto.

El sistema Sirius es cada vez más utilizado y estudiado en la literatura, tanto en el entorno móviles (Luna et al., 2015), como en sitios web en función del tipo de página (Torrente et al., 2013) o de cara a mejorar la experiencia de usuario y su medición (Bañón-Gomis et al., 2013). En nuestro estudio hemos decidido aplicar el sistema Sirius para el análisis de la usabilidad de las principales marcas de empresas de moda españolas. Para ello vamos a utilizar datos de Interbrand como referencia en nuestro estudio.

\section{Resultados}

La consultora Interbrand ${ }^{7}$ fue fundada en 1974 por John Murphy como Novamark en el Reino Unido. En 1979 Novamark abre una oficina en EE.UU bajo el nombre Interbrand, pasando de enfocarse al registro de marcas y elección de denominaciones a hacerlo en la estrategia de marca y su diseño. Tras ser comprada por el grupo Omnicom en 1993 su crecimiento internacional se hace más rápido todavía.

Interbrand es la creadora del informe Best Global Brands ${ }^{8}$ en el cual analizan de manera anual las marcas globales más valiosas del mundo. La metodología de valoración desarrollada por Interbrand cumple la norma ISO y para calcular el valor de una marca utiliza criterios como el rendimiento financiero de los productos o servicios, el impacto de la marca a la hora de influir en la toma de decisiones del consumidor así como la solidez de la marca en garantizar ingresos a la compañía poseedora de la misma o en

\footnotetext{
7 Interbrand es una consultora especializada en el estudio de las marcas en 27 países de todo el mundo, entre ellos España. 8 http://interbrand.com/best-brands/best-global-brands/2016/
} 
permitir solicitar un precio superior por los productos o servicios. Se pueden encontrar en su web datos de la clasificación desde el año 2000, fecha en que el ranking comprendía 75 marcas.

En España establece un ranking de las mejores marcas españolas siguiendo los criterios marcados por la norma ISO $10668^{9}$. La clasificación española es realizada bianualmente, siendo la última en 2015.

Por lo tanto gracias al estudio de Interbrand podemos disponer de los datos y la referencia que nos permita relacionar el valor de marca con la usabilidad, apoyados en la metodología Sirius explicada anteriormente. Para ello se han analizado las heurísticas propuestas por Suárez (2011) sobre las principales marcas españolas de moda, según Interbrand (2015), obteniendo un nuevo ranking de usabilidad.

El valor que se obtiene en el análisis de usabilidad está reflejado en términos de porcentaje. Siendo 0\% el valor más bajo y el 100\% el valor más alto que se puede obtener. Asimismo, Suárez establece una relación directa de estos resultados con el sistema de calificación habitual de cero a diez puntos.

\begin{tabular}{|c|c|c|c|}
\hline \multicolumn{2}{|c|}{$\begin{array}{l}\text { Mejores marcas españolas } \\
\text { INTERBRAND }\end{array}$} & \multirow{2}{*}{$\begin{array}{l}\text { Cálculo } \\
\text { usabilidad } \\
82,91\end{array}$} & \multirow{2}{*}{$\begin{array}{l}\text { Posición } \\
\text { usabilidad } \\
5\end{array}$} \\
\hline 1 & Zara & & \\
\hline 2 & Bershka & 82,00 & 6 \\
\hline 3 & Mango & 93,59 & 1 \\
\hline 4 & Massimo Dutti & 91,60 & 3 \\
\hline 5 & El Corte Inglés & 89,08 & 4 \\
\hline 6 & Desigual & 92,26 & 2 \\
\hline
\end{tabular}

Fuente: Rodríguez, 2016

9 En la valoración de una marca monetaria la norma ISO 10668 tiene en cuenta los datos de mercado y financieros de la marca, los aspectos de comportamiento de la misma, los aspectos legales del mercado y entorno en el que se encuentra la marca, y el uso de los datos y los supuestos de calidad con lo que trabaja esta marca.
Sobre cada una de las marcas se han evaluado una serie de heurísticas agrupadas en diez elementos generales. De estas heurísticas se ha obtenido una media que puede alcanzar un análisis más pormenorizado de los elementos a evaluar.

Vamos a ver el detalle del análisis realizado para la web de una de las marcas, concretamente Zara. En primer lugar vamos a ver los datos generales de la evaluación.

\begin{tabular}{|l|l|}
\hline Evaluador & Iria Rodríguez Pouso \\
\hline Fecha de evaluación & $10 / 6 / 15$ \\
\hline Nombre del sitio evaluado & Zara \\
\hline URL del sitio evaluado & http://www.zara.com/es/ \\
\hline Tipo de sitio evaluado & Comercio electrónico \\
\hline $\begin{array}{l}\text { Navegador con el que se } \\
\text { revisa }\end{array}$ & Chrome \\
\hline Versión del navegador & $43.0 .2357 .81 \mathrm{~m}$ \\
\hline
\end{tabular}

Las leyendas de los códigos utilizados son las siguientes

- Valor de 0 a 10: No se cumple en absoluto $=0$; Se cumple totalmente $=10$

- NA: Criterio no aplicable en el sitio

- NTS: No se cumple en todo el sitio

- NEP: No se cumple en los enlaces principales

- NPP: No se cumple en la página principal

- NPI: No se cumple en alguna página interior

- S: Se cumple el criterio

Analizamos los criterios del heurístico «Aspectos Generales» que comprende elementos relacionados con los objetivos del sitio, la apariencia (o look\&feel), coherencia y nivel de actualización de contenidos. 


\begin{tabular}{|c|l|c|}
\hline Código & Criterio & Valor \\
\hline AG1 & Objetivos del sitio web concretos y bien definidos & 7 \\
\hline AG2 & Contenidos y servicios ofrecidos precisos y completos & 10 \\
\hline AG3 & Estructura general del sitio web orientada al usuario & 6 \\
\hline AG4 & Look \& Feel general se corresponde con los objetivos, características, contenidos y servicios del sitio web & 9 \\
\hline AG5 & Diseño general del sitio web reconocible & 9 \\
\hline AG6 & Diseño general del sitio web coherente & 9 \\
\hline AG8 & Se utiliza el idioma del usuario & Se da soporte a otro/s idioma/s \\
\hline AG9 & Traducción del sitio completa y correcta & S \\
\hline AG10 & Sitio web actualizado periódicamente & S \\
\hline
\end{tabular}

El tercer criterio es «Estructura y Navegación» y tiene en cuenta elementos relacionados con la idoneidad de la arquitectura de la información y la navegación del sitio.

\begin{tabular}{|c|c|c|}
\hline Código & Criterio & Valor \\
\hline EN.1 & Se ha evitado pantalla de bienvenida & NPP \\
\hline EN.2 & Estructura de organización y navegación adecuada & 6 \\
\hline EN.3 & Organización de elementos consistente con las convenciones & 6 \\
\hline EN.4 & Control del número de elementos y de términos por elemento en los menús de navegación & 5 \\
\hline EN.5 & Equilibrio entre profundidad y anchura en el caso de estructura jerárquica & S \\
\hline EN.6 & Enlaces fácilmente reconocibles como tales & NTS \\
\hline EN.7 & La caracterización de los enlaces indica su estado (visitados, activos) & NTS \\
\hline EN.8 & No hay redundancia de enlaces & S \\
\hline EN.9 & No hay enlaces rotos & S \\
\hline EN.10 & No hay enlaces que lleven a la misma página que se está visualizando & S \\
\hline EN.11 & En las imágenes de enlace se indica el contenido al que se va a acceder & S \\
\hline EN.12 & Existe un enlace para volver al inicio en cada página & S \\
\hline EN.13 & $\begin{array}{l}\text { Existen elementos de navegación que orienten al usuario acerca de dónde está y cómo deshacer } \\
\text { su navegación (ej: migas) }\end{array}$ & S \\
\hline EN.14 & Existe mapa del sitio para acceder directamente a los contenidos sin navegar & NTS \\
\hline
\end{tabular}


El «Control y Retroalimentación» analiza elementos relacionados con la libertar del usuario en la navegación y la información proporcionada al mismo en el proceso de interacción con el sitio web.

\begin{tabular}{|c|l|c|}
\hline Código & Criterio & Valor \\
\hline CR.1 & El usuario tiene todo el control sobre la interfaz & S \\
\hline CR.2 & Se informa al usuario acerca de lo que está pasando & S \\
\hline CR.3 & Se informa al usuario de lo que ha pasado & S \\
\hline CR.4 & Existen sistemas de validación antes de que el usuario envíe información para tratar de evitar errores & S \\
\hline CR.5 & Cuando se produce un error, se informa de forma clara y no alarmista al usuario de lo ocurrido y de & S \\
\hline CR.6 & Cómo solucionar el problema & Se ha controlado el tiempo derespuesta \\
\hline CR.7 & Se ha evitado que las ventanas del sitio anulen o se superpongan a la del navegador \\
\hline CR.8 & Se ha evitado la proliferación de ventanas en la pantalla del usuario & S \\
\hline CR.9 & Se ha evitado la descarga por parte del usuario de plugins adicionales \\
\hline CR.10 & Si existen tareas de varios pasos, se indica al usuario en cual está y cuantos faltan para completar la tarea & S \\
\hline
\end{tabular}

El heurístico de «Elementos Multimedia» analiza elementos relacionados con el grado de adecuación de los contenidos multimedia al sitio web.

\begin{tabular}{|c|l|c|}
\hline Código & Criterio & Valor \\
\hline EM.1 & Fotografías bien recortadas & S \\
\hline EM.2 & Fotografías comprensibles & S \\
\hline EM.3 & Fotografías con correcta resolución & S \\
\hline EM.4 & El uso de imágenes o animaciones proporciona algún tipo de valor añadido & S \\
\hline EM.5 & Se ha evitado el uso de animaciones cíclicas & S \\
\hline EM.6 & El uso de sonido proporciona algún tipo de valor añadido & NA \\
\hline
\end{tabular}

El heurístico «Búsqueda» se refiere a elementos relacionados con el buscador implementado en el sitio web.

\begin{tabular}{|l|l|l|}
\hline Código & Criterio & Valor \\
\hline BU.1 & La búsqueda, si es necesaria, se encuentra accesible desde todas las páginas del sitio & S \\
\hline BU.2 & Es fácilmente reconocible como tal & 7 \\
\hline BU.3 & Se encuentra fácilmente accesible & 10 \\
\hline BU.4 & La caja de texto es lo suficientemente ancha & 10 \\
\hline BU.5 & Sistema de búsqueda simple y claro & 10 \\
\hline BU.6 & Permite la búsqueda avanzada & 0 \\
\hline BU.7 & Muestra los resultados de la búsqueda de forma comprensible para el usuario & 8 \\
\hline BU.8 & Asiste al usuario en caso de no poder ofrecer resultados para una consultada dada & 0 \\
\hline
\end{tabular}


Finalmente el heurístico «Ayuda» se refiere a elementos relacionados con la ayuda ofrecida al usuario durante la navegación por el sitio.

\begin{tabular}{|l|l|c|}
\hline Código & Criterio & Valor \\
\hline AY.1 & El enlace a la sección de Ayuda está colocado en una zona visible y estándar & NTS \\
\hline AY.2 & Fácil acceso y retorno al/del sistema de ayuda & 0 \\
\hline AY.3 & Se ofrece ayuda contextual en tareas complejas & NEP \\
\hline AY.4 & FAQs (si las hay) correcta la elección como la redacción de las preguntas & 0 \\
\hline AY.5 & FAQs (si las hay) correcta la redacción de las respuestas & 0 \\
\hline
\end{tabular}

Tenemos en cuenta ahora la relevancia del incumplimiento de los criterios heurísticos. Por ejemplo para «Aspectos Generales» y el caso de una web de comercio electrónico llegamos a la siguiente tabla.

\begin{tabular}{|l|l|l|}
\hline Código & Criterio & Relevancia \\
\hline AG1 & Objetivos del sitio web concretos y bien definidos & MA \\
\hline AG2 & Contenidos y servicios ofrecidos precisos y completos & CR \\
\hline AG3 & Estructura general del sitio web orientada al usuario & MA \\
\hline AG4 & Look \& Feel general se corresponde con los objetivos, características, contenidos y servicios del sitio web & ME \\
\hline AG5 & Diseño general del sitio web reconocible & MA \\
\hline AG6 & Diseño general del sitio web coherente & MA \\
\hline AG7 & Se utiliza el idioma del usuario & MA \\
\hline AG8 & Se da soporte a otro/s idioma/s & MA \\
\hline AG9 & Traducción del sitio completa y correcta & MA \\
\hline AG10 & Sitio web actualizado periódicamente & MA \\
\hline
\end{tabular}

En este caso no vamos a incluir las tablas para todos los heurísticos. Lo que sí es importante es conocer el criterio de valoración de los códigos. Para los códigos de valoración de los heurísticos correlacionamos con valores numéricos de la siguiente manera.

\begin{tabular}{|c|c|c|c|}
\hline NTS & 0 & Y para los de relevancia \\
\hline NEP & 2,5 & \\
\hline NPP & 5 & CR & 8 \\
\hline NPI & 7,5 & MA & 4 \\
\hline S & 10 & ME & 2 \\
\hline $0-10$ & $0-10$ & MO & 1 \\
\hline NA & 0 & & 4 \\
\hline
\end{tabular}


Para cada uno de los valores anteriores se ha tenido en cuenta también aspectos como la relevancia, el valor interno (vc) y el valor interno de la relevancia (rc). Con esto se puede calcular un factor de corrección (fc). Llegados a este punto ya podemos calcular un porcentaje de usabilidad. Este cálculo se basa en los criterios evaluados a partir de las siguientes fórmulas.

Porcentaje de Usabilidad (PU)

$$
P U=\frac{\sum_{i=1}^{i=n c e}(f c i * v c i)}{\sum_{\substack{i=1 \\ i=1}}^{i=1}(f c i * 10)} * 100
$$

Factor de Corrección aplicado a los criterios evaluados (fc)

$$
f c i=\frac{r c i}{\sum_{j=1}^{j=n c e} r c j}
$$

Los datos para el cálculo son los siguientes

\begin{tabular}{|l|r|}
\hline No de criterios evaluados & 79 \\
\hline $\begin{array}{l}\text { Sumatorio de los valores de } \\
\text { relevancia de los criterios evaluados }\end{array}$ \\
\hline $\begin{array}{l}\text { Sumatorio de la columna y de cada } \\
\text { criterio }\end{array}$ & 326 \\
\hline $\begin{array}{l}\text { Sumatorio de la columna } \mathbf{h} \text { *10 de } \\
\text { cada criterio }\end{array}$ & 10,2914110429 \\
\hline
\end{tabular}

En el caso de los NA no se les aplica relevancia (segunda fila) ni se tienen en cuenta (tercera y cuarta filas). Otros detalles de la fórmula:

- nce: número de criterios evaluados. Los NA no contabilizan.

- vc: valor de evaluación de un criterio (campo de la columna $\mathrm{f}$ «Valor interno» de cada criterio)

- fc: Factor de corrección aplicado al criterio evaluado

Para el cálculo del valor del factor de corrección de cada uno de los criterios evaluados se debe tener en cuenta también el valor de relevancia
- rc: Valor de relevancia que corresponde a un criterio determinado (Valor Interno de Relevancia)

Para el caso que nos ocupa el porcentaje de usabilidad de Zara es del 82.91\%.

Realizando el mismo análisis para el resto de marcas obteníamos la tabla anterior en la que podíamos comprobar como según Interbrand Zara era la marca número uno por valor de marca mientras que era la número 5 de 6 por porcentaje de usabilidad. El detalle del análisis para cada marca sería el siguiente. (Tabla 2)

De estos resultados, se observa que los aspectos mejor cuidados son «aspectos generales», «control y realimentación», «entendibilidad y facilidad en la interacción» y «elementos multimedia» de las páginas web. Asimismo, los aspectos menos valorados son la «búsqueda» y la «ayuda» en las páginas web.

Estos aspectos menos tenidos en cuenta a la hora de diseñar páginas web, implica que algunas marcas con un muy buen índice de usabilidad en el resto de aspectos bajen su media, por no cuidar todos los elementos que hacen sencilla la navegación para los usuarios. Además en el caso de páginas de comercio electrónico la función de búsqueda es de vital importancia para activar el proceso de conversión a ventas.

Si se analizan estos aspectos de forma diferenciada por marca, se observa que los que muestran grandes picos diferenciales son «control y realimentación» $y$ «entendibilidad y facilidad en la interacción» para el caso de los mejor cuidados, de tal modo que aunque forman parte de una buena práctica de usabilidad, no todas las marcas cuidan este aspecto del mismo modo. Los elementos multimedia se muestran claramente importantes al mismo nivel para todas las marcas. En el caso de los elementos menos 


\section{Tabla 2 • Evaluación de los aspectos de usabilidad de las Mejores Marcas españolas 2015}

\begin{tabular}{|c|c|c|c|c|c|c|c|c|c|c|}
\hline & 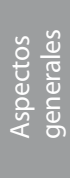 & 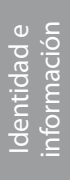 & 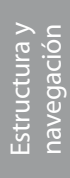 & $\begin{array}{l}\frac{0}{0} \\
\frac{\pi}{2} \\
\frac{7}{0} \\
\propto\end{array}$ & 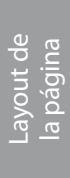 & 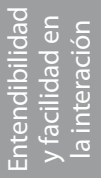 & 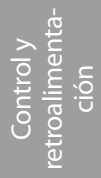 &  & $\begin{array}{l}\frac{\pi}{8} \\
\frac{d}{2} \\
\frac{\tilde{\sigma}}{5} \\
\frac{\pi}{0}\end{array}$ & $\frac{\pi}{\frac{\pi}{2}}$ \\
\hline Zara & 9 & 8,5 & 6,57 & 10 & 8,45 & 10 & 10 & 10 & 6,87 & 0,5 \\
\hline Bershka & 9,7 & 10 & 7,5 & 6,67 & 8,5 & 8,57 & 9 & 10 & 7,12 & 0 \\
\hline Mango & 9,7 & 8 & 7,93 & 9,08 & 9 & 10 & 10 & 10 & 8,75 & 10 \\
\hline $\begin{array}{l}\text { Massimo } \\
\text { Dutti }\end{array}$ & 9,8 & 10 & 8,57 & 8,33 & 8,5 & 10 & 9,75 & 10 & 7 & 10 \\
\hline Desigual & 9,7 & 9,67 & 8,28 & 9,67 & 8,5 & 10 & 9 & 10 & 7,25 & 9,5 \\
\hline Media & 9,58 & 9,23 & 7,77 & 8,75 & 8,59 & 9,71 & 9,55 & 10 & 7,4 & 6 \\
\hline
\end{tabular}

Fuente: Rodríguez, 2016

cuidados, la búsqueda muestra menos picos di- de Zara y Bershka en cuanto a la poca valoraferenciales en su análisis que la ayuda. El caso ción del heurístico «ayuda» es notable.

\section{Figura 2 - Elementos mejor cuidados en usabilidad digital}

\section{Elementos mejor cuidados}

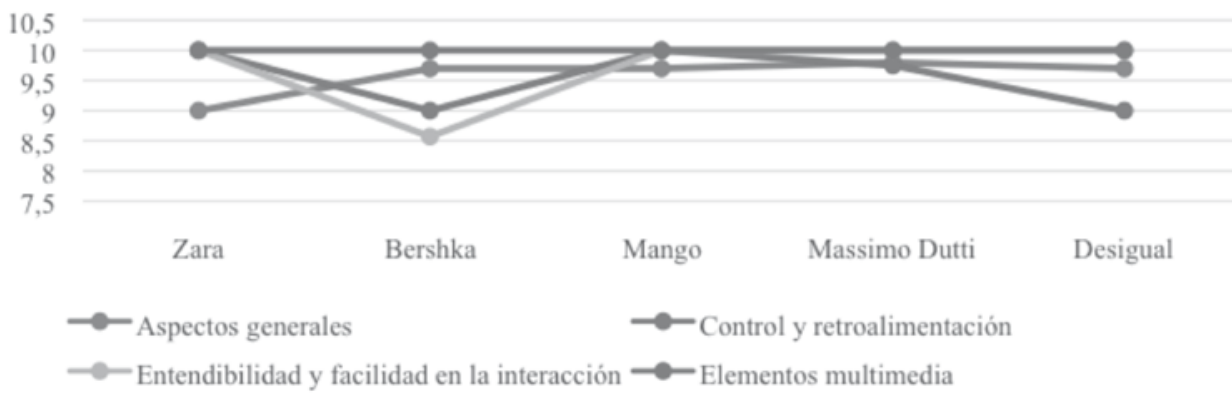




\section{Figura 3 - Elementos menos cuidados en usabilidad digital}

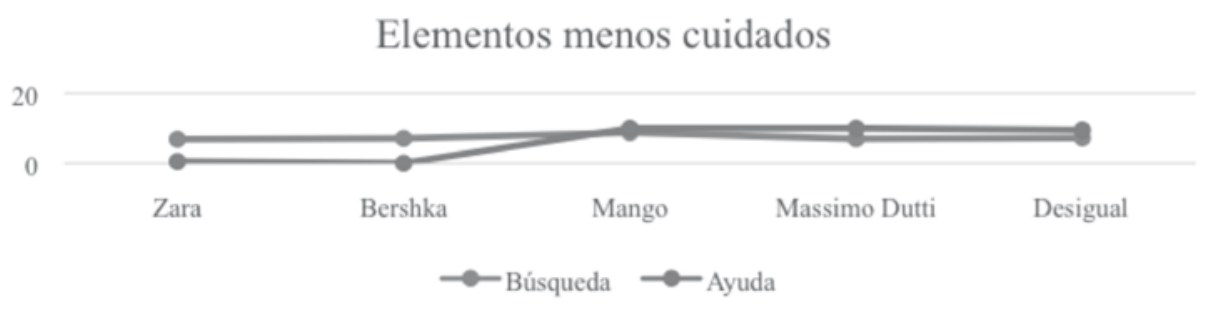

\section{Conclusiones}

La usabilidad web representa una disciplina cada vez más importante y creciente. Las marcas se comunican cada vez más con sus audiencias mediante sus páginas web, por lo que la usabilidad como aspecto relevante de dicha interacción debe ser medida para poder ser mejorada y alineada con los objetivos de cada tipo de página web.

La evaluación heurística, como método de evaluación de usabilidad, es uno de los métodos más fiables para dicho proceso de medición. No solo por el desarrollo y evolución que se ha visto reflejado por varios de los estudiosos y analistas de esta disciplina, sino también porque da la posibilidad de llevarlo a cabo sin usuarios, de realizar el análisis en las diferentes etapas de un producto digital, y de poder centrarse, durante la evaluación, en un aparatado de la interfaz o en todos ellos. Todo esto facilita detectar errores e introducir mejoras en las aplicaciones digitales.

Desde el punto de vista de las aplicaciones digitales, la usabilidad trata de que éstas puedan lograr los objetivos para los que fueron desarro- lladas, tanto desde la funcionalidad, como la forma en la que los usuarios son capaces de llevar a cabo las tareas que implica, por lo tanto, la rapidez en el aprendizaje, la eficiencia de uso o la propensión a cometer errores son temas a analizar de cara a la optimización de la usabilidad.

Las marcas de moda estudiadas no solo son un referente en los mercados españoles sino también a nivel mundial. Era esperable por lo tanto que obtuvieran en general una usabilidad alta en sus páginas web principales. La elección de las mismas se debe a que de este modo es más fácil detectar los errores comunes en usabilidad, sin incluir información que pueda distorsionar los datos con el uso de marcas menos punteras tecnológicamente. Pero así mismo hemos podido comprobar aspectos relevantes y grandes diferencias entre las mismas.

Por ejemplo los elementos de búsqueda y ayuda, que están siendo cada vez más omitidos en el diseño y desarrollo de las páginas web o aplicaciones digitales en general, aparecen claramente con una valoración muy baja en marcas 
como Zara o Bershka. Por otra parte es importante contextualizar estos hechos con el entorno. Se da por supuesto que los usuarios son cada vez más avanzados en competencias digitales y que conocen el uso y manejo de todo tipo de aplicaciones. Sin embargo y aunque se utilicen los estándares comunes en los desarrollos se debe tener en cuenta que cada usuario puede tener intencionalidades diferentes en cada web, por lo que omitir estas zonas puede llegar a implicar en muchos casos pérdidas, tanto de interacción como de negocio.
Siguientes pasos en nuestra investigación serán analizar los motivos de estas diferencias. También hemos abierto el camino para desarrollar un estudio de mayor calado teniendo en cuenta las principales marcas españolas según Interbrand, buscando correlacionar la usabilidad web de dichas marcas con el impacto de la misma en el valor de marca. Así mismo el creciente uso de dispositivos móviles nos aconseja también ampliar el estudio en esa línea, que puede tener a su vez relación con el impacto en el uso de elementos de búsqueda y ayuda. 


\section{Bibliografía}

Abran, A.; Khelifi, A.; Suryn, W. y Seffah, A. (2003). Usability meanings and interpretations in ISO standards. Software Quality Journal, 11, 4, 325-338.

Bañón-Gomis, A., Tomás-Miquel, J. V., y Expósito-Langa, M. (2014). Improving User Experience: A Methodology Proposal for Web Usability Measurement. In Strategies in EBusiness (123-145). Springer US.

Berners-Lee, T. (2013). The power of the Web is in its universality. Access by everyone regardless of disability is an essential aspect. En World Wide Web Consortium (W3C). Disponible en: http://www.w3.org/standards/webdesign/ accessibility

Bevan, N. (1997). Usability issues in web site design. In HCI, vol. 2, 803-806.

Bevan, N. (1997). Quality and usability: a new framework. Achieving software product quality, 25-34.

Bevan, N., \& Macleod, M. (1994). Usability measurement in context. Behaviour \& information technology, 13(1-2), 132-145.

Borrero, B. (2014). Unos lideran, otros siguen. En Mejores Marcas Españolas 2013. Disponible en: http://interbrand. com/wp-content/uploads/2015/08/Interbrand-Best-Spanish-Brands-2013.pdf

British Standards Institute Staff. (1916). Medical devices. Guidance on the Application of Usability Engineering to Medical Devices. Londres: B S I Standars.

Calvo-Fernández et al. (2013). Métodos de evaluación con Usuarios. Universitat Oberta de Catalunya.

Card, S. K.; Moran, T. P.; Newell, A. (1983). The psychology of human-computer interaction. Hillsdale: Lawrence Erlbaum Associates.

D'Hertefelt, S. (2000). Emerging and future usability challenges: designing user experiences and user communities. En InteractionArchitect.com. Disponible en: http://users.skynet.be/fa250900/future/vision20000202shd.htm

Dix, A., Finlay, J., Abowd, G. y Beale, R. (1993). HumanComputer Interaction, Englewood Cliffs, NJ, PrenticeHall.

Garrett, J. J. (2000). Los Elementos de la Experiencia de Usuario. En jjg.net. disponible en: http://www.jjg.net/elements/translations/elements_es.pdf
Garvin, D. A. (1984). What does «product quality» really Mean?. Sloan management review, 25.

Hassan, Y. (2006). Factores del Diseño Web Orientado a la Satisfacción y No-Frustración de Uso. En Revista Española de Documentación Científica. 29, 2, Abril-Junio, 239-257.

Hassan, Y. y Martin, F. J. (2004). Propuesta de adaptación de la metodología de diseño centrado en el usuario para el desarrollo de sitio web accesibles. En Revista Española de Documentación Científica. 27, 3, 330-344.

Hassan, Y. y Ortega, S. (2009). Informe APEI sobre usabilidad. Gijón: Asociación Profesional de Especialistas en Información.

Islam, M. N. y Bouwman, H. (2016). Towards user-intuitive web interface sign design and evaluation: A semiotic framework. International Journal of Human-Computer Studies, vol. 86, 121-137.

Interbrand. (2016). Mejores Marcas Españolas 2015. Disponible en: http://www.mejoresmarcasinterbrand.com/

ISO 9241-171. (2008). Ergonomics of human-system interaction. Switzerland: International Organization for Standardization.

ISO 10668. (2010). Brand valuation - Requirements for monetary brand valuation. Switzerland: International Organization for Standardization.

Jordan, P. W., Thomas, B., McClelland, I. L., y Weerdmeester, B. (Eds.). (1996). Usability evaluation in industry. CRC Press.

Lee, D.; Junghoon M.; Kim, Y.J.; Mun, Y.Y. (2015) Antecedents and consequences of mobile phone usability: Linking simplicity and interactivity to satisfaction, trust, and brand loyalty. Information \& Management vol. 52, no 3, 295-304.

Lewis, C.; Polson, P.; Wharton, C.; Rieman, J. (1990). Testing a walkthrough methodology for theory-based design of walk-up-and-use interfaces. En Proceedings of ACM CHI'90. 1-5 de abril, 235-242.

Lewis, C.; Polson, P.; Wharton, C.; Rieman, J. (1994). The cognitive walkthrough method: a practitioner's guide. En Usability Inspection Method (pp. 105 - 140). Nueva York: John Wiley and Sons.

Luna, H., Mendoza, R., Vargas, M., Munoz, J., Alvarez, F. J., $\&$ Rodriguez, L. C. (2015). Using Design Patterns as Usabi- 
lity Heuristics for Mobile Groupware Systems. IEEE Latin America Transactions, vol. 13, n 12, 4004-4010.

Moreno, A. (2003). Diseño centrado en el usuario (I). Introducción. El profesional de la información. vol. 12, nº 1, 52 - 52 .

Morville, P. (2004) User Experience Design. En Semantic Studios. Disponible en: http://semanticstudios.com/publications/semantics/000029.php

Nielsen, J. (1993). Usability engineering. Nueva York: Academic Press.

Nielsen, J. (1994). Usability engineering. Boston: Academic Press.

Nielsen, J. (1995). 10 Usability Heuristics for User Interface Design. En Nielsen Norman Group. Disponible en: https:// www.nngroup.com/articles/ten-usability-heuristics/

Nielsen, J. (1999). Usability engineering at a discount. En: G. Salvendy; M. J. Smith (eds.). Designing and using humancomputer interfaces and knowledge based systems. Ámsterdam: Elsevier Science Publishers.

Nielsen, J. (2003). Usability 101: Introduction to Usability. En UseIt.com Alertbox. Disponible en: http://www.useit. com/alertbox/20030825.html

Nielsen, J. (2009) IA Task Failures Remain Costly. En UseIt. com Alertbox. Disponible en: http://www.useit.com/alertbox/ia-failures.html

Nielsen, J. y Loranger, H. (2006). Usabilidad. Prioridad en el diseño web. Madrid: Anaya Multimedia.

Nielsen, J y Mack, R. (1994). Usability inspection methods. Nueva York: John Wiley and Sons.

Nielsen, J. y Molich, R. (1990). Heuristic evaluation of user interfaces. Proc. ACM CHI'90 Conf. Seattle, WA, 1-5 April, 249-256

Ortega, S. (2013). Introducción a la usabilidad y su evaluación. Universitat Oberta de Catalunya.

Pearl, J. (1984). Heuristics: intelligent search strategies for computer problem solving. Londres: Addison-Wesley Publ. Co.
Perurena, L. y Moráguez, M., (2013). «Usabilidad de los sitios Web, los métodos y las técnicas para la evaluación» en Revista Cubana de Información en Ciencias de la Salud [En Línea] Vol. 24. No. 2. Abril-junio 2013, Universidad de La Habana

Polson, P.G. y Lewis, C.H. (1990). Theory-based design for easily learned interfaces. En Human-Computer Interaction. 5 191-220.

Rodríguez, I. (2016). La competitividad de las marcas españolas: el diseño integrado de la usabilidad digital. Tesis de doctorado no publicada, Universidad Antonio de Nebrija, Facultad de Ciencias de la Comunicación, Madrid.

Rowley, D y Rhoades, D. (1992). «The cognitive jogthrough: A fast-paced user interface evaluation procedure». En Proceedings of ACM CHI '92. 3-7 de mayo, 389-395.

Suárez, M. C. (2011). SIRIUS: Sistema de Evaluación de la Usabilidad Web Orientado al Usuario y basado en la Determinación de Tareas Críticas. Tesis doctoral. Oviedo: Departamento de Informática, Universidad de Oviedo.

Torrente, M. C. S., Prieto, A. B. M., GutiéRrez, D. A., \& De Sagastegui, M. E. A. (2013). Sirius: A heuristic-based framework for measuring web usability adapted to the type of website. Journal of Systems and Software, vol. 86, $\mathrm{n}^{\circ}$ 3, 649-663.

Wixon, D. y Wilson, C. (1997). The usability-engineering framework for product design and evaluation. En Handbook of human computer interaction (pp. 653-688). Amsterdam: Elsevier Science.

Woodward, B. (1998). Evaluation methods in usability Testing CS5326.

Yu, N., \& Kong, J. (2016). User experience with web browsing on small screens: Experimental investigations of mobile-page interface design and homepage design for news websites. Information Sciences, vol. 330, 427-443.

Zapata, M. (2013). Métodos de evaluación sin usuarios. Universitat Oberta de Catalunya. 